26. Lin, P.C., Tobin, K.D., Grumet, M. \& Lin, S. J. Cell Biol. 84, 455 (1980)

27. MacLean-Fletcher, s. \& Pllard, T.D Cell 20, $329(1980)$

28. Hartwig, J.H. \& Stossel, T.P. J. molec. Biol. 134, 539 (1979).

29. Morris, A. \& Tannenbaum, J. Narure 287, 637 (1980),

30. Selden, L.A., Gershman, L.C. \& Estes, J.E. Biochem. biophys. Res. Commun. 95, 1854 (1980).

11. Grumet, M. \& Lin, S. Cell 21, 439 (1980).

32. Isenberg, G., Aebi,U. \& Pollard, T.D. Nature this issue, p. 455 .

33. Grumet, M., Loonsk, J. \& Lin, S. J. Cell Biol. 87, 217a (1980)

34. Lin, D.C. \& Lin, S. J. Cell Biol. 87, 213a (1980).

35. Yin, H.L. \& Stossel, T.P. Nature 281, 583 (1979).
36. MacLean-Fletcher, S. \& Pollard, T.D. J. Cell Biol, 85, 414 (1980).

37. Pollard T D , Levy, J., Isenberg, G. \& Aebi, U. J Cell Biol. 87, 223a (1980).

38. MacLean-Fletcher, S. \& Pollard, T.D. Biochem. biophys. Res. Commun. 96, 18$) 1980$ ).

39. Hitchcock, S.E., Carlsson, L. \& Lindberg, U. Cell 7, 531 (1976).

40. Chaponnier, C., Borgia, R., Rungger-Brăndle, E., Weil, R. \& Gabbiani, G. Experientia 35, 1039 (1979).

Norberg, R., Thorstensson, R., Utter, G. \& Fagraeus, A Eur. J. Biochem. 100, 575 (1979).

42. Harris, H.E. \& Weeds, A.G. Cell Biol. Int. Reports 4, 741 (1980)

43. Brown, S.S. \& Spudich, J.A. J. Cell Biol. 87,224a (1980).

\title{
Where do cosmic rays come from?
}

from a Correspondent

ABOUT once a second a single atomic nucleus carrying on its own at last 10 Joules of energy from Space hits an air nucleus in the Earth's atmosphere. It thereby initiates an enormous shower of secondary particles which builds up to an extensive air shower (EAS) of $10^{10}$ particles by the time it reaches sea-level. Such a primary cosmic ray particle has been accelerated somewhere in the Universe through the equivalent of a voltage difference of $6 \times 10^{19}$ volts. How and where this acceleration takes place remain among the major unsolved questions of high energy astrophysics.

The origin of cosmic ray particles has been a subject of controversy for more than 50 years and has resulted in something of an East-West divide amongst astrophysicists. On the one side V. L. Ginzburg (P. N. Lebedev Institute of Physics, Academy of Science of the USSR, Moscow) and others have argued consistently that these cosmic ray nuclei originate mainly in our own Galaxy. On the other side, British theoretical astronomers, such as G. R. Burbridge (University of California), have maintained that the sources must be extragalactic. Whichever viewpoint is held, however, there is general agreement that supernovae explosions play a significant part in cosmic ray particle acceleration.

Most cosmic rays, of course, have energies far less than the highest energy particles; the spectrum at the Earth peaks at energies of $10^{9} \mathrm{eV}$ per nucleus and falls rapidly to energies greater than $10^{20} \mathrm{eV}$ per nucleus ( $1 \mathrm{ev}=1.6 \times 10^{-19}$ Joules). At the highest energies ( $>10^{18} \mathrm{eV}$ per nucleus) astrophysicists have so far failed to produce a satisfactory model for the acceleration mechanism. Almost by a process of elimination, however, the net seems to be closing in on possible source regions. Briefly the case is as follows. The ultra-high energy particles cannot originate and remain captured in the Galactic disc because the Galactic magnetic field is not strong or extensive enough to contain the particles. For a Galactic origin it is generally thought that strong anisotropy in the particle arrival directions should exist - presumably from the centre of the Galaxy. Again, the particles cannot come from the deep depths of space. They could not travel that far without colliding with the Universal $2.7^{\circ} \mathrm{K}$ microwave background photons and undergoing strong absorption through pion production at energies greater than $10^{19} \mathrm{eV}$ per nucleus. The experimental evidence shows that rather than a cut-off in the cosmic ray energy spectrum occurring at $10^{19} \mathrm{eV}$, it is just at this point that a flattening of the spectrum appears to occur. So where does that leave us?

The latest East-West confrontation took place recently in the beautiful old Russian captial of Leningrad where the VIIth European Cosmic Ray Symposium was held from September 15th-19th. Here A. W. Wolfendale (University of Durham) suggested that the problem could be resolved by a model in which those ultrahigh energy particles reaching the Earth come predominantly from the nearby local supercluster of galaxies in Virgo. The actual sources would be active galaxies within the cluster of 2,500 galaxies. Wolfendale and his colleagues have developed their model to explain the available experimental data and it predicts an obvious anisotropy in the particles arrival direction at the Earth. Such an anisotropy in the general direction of Virgo is indeed observed for the highest energy particles detected by the Haverah Park EAS Detector Array in Yorkshire (16th Int. Cosmic Ray Conf. Kyoto 13, 130; 1979.

At Leningrad J. Lloyd-Evans (University of Leeds) presented the latest data on the anisotropy analysis from the Haverah Park Group. These indeed confirms a sharp and significant change in the phase of preferred arrival direction from low Galactic latitudes to high positive latitudes as the primary energy increases above $10^{19} \mathrm{eV}$ in EAS detected at Haverah Park. This change occurs sharply at $10^{19} \mathrm{eV}$ per nucleus, just where the flattening in the energy spectrum is observed. So the Wolfendale model fits in well with the latest experimental data.

However, the 'extragalactic' Western scientists were not allowed to have it all their own way in Leningrad. D. D. Krasilnikov (Inst. of Cosmophysical Research and Astronomy, Yakutsk, USSR), presented a paper entitled

'Intensity Anisotropy of Highest Energy Cosmic Rays, its Energy Dependence'. In this the author considers the distribution on the celestial sphere of all the 313 cosmic ray particles with energies above $10^{19} \mathrm{eV}$ detected by the four large EAS detector arrays in England, Australia, US and the USSR. Krasilnikov too demonstrates that the anisotropy increases and changes phase as the primary energy increases. In particular, he selects out for special treatment the particles with energy/ $3 \times 10_{19} \mathrm{eV} /$ nucleus. He splits the data in half in four different ways: (a) those coming from below and those above the Galactic equaltorial belt of $\pm 30^{\circ}$ latitude; (b) those coming from inside and those outside the Galactic equatorial belt of \pm $30^{\circ}$ from the Galactic centre; (c) those coming from opposite hemispheres pointing towards and away from the Galactic centre; and (d) those coming from opposite hemispheres pointing towards and away from the centre of the Virgo cluster. Taking the ratios of the intensities from the two groups in each of the four cases, Krasinilkov finds that only in case (c) does the intensity ratio $(=0.43 \pm 0 ; 14)$ differ significantly from unity. Thus he claims the measured anisotropy is associated with Galactic co-ordinates. The author therefore concludes that these results point to a Galactic source for the particles and infer a mass composition of heavy or middle mass nuclei and a modulation of their propagation directions by Galactic disc and halo magnetic fields. $\mathrm{He}$ argues that although supernovae can only directly accelerate the particles up to $\sim 10^{17} \mathrm{eV}$ per nucleus, further acceleration can be achieved by the magnetic fields of the Galaxy, both disc and halo, over time scales of $10^{7}$ to $10^{8}$ years.

Significantly, Krasilnikov needs heavy primary nuclei to support his theory. Unfortunately, as yet, there is little experimental guide as to the primary mass composition at these energies. What there is suggests that protons are still abundantly present as at lower energies. The East $v$ West battle is thus still alive. We must now await the next round, to take place at the 17th IUPAP Conference in Paris next July.

The Leningrad symposium was, not unexpectedly, dominated by the Russians, since they combined the biannual European Symposium with an annual national cosmic ray meeting. The great magnitude of the Russian effort showed up forcefully in the special workshop session devoted to cosmogenic nuclides - that is the study of individual nuclear isotope concentration in meteorites. The analysis and interpretation of such data is now reaching a very high degree of sophistication.

ERRATUM
In the News and Views article "Lessons from a
peptidergic neurone" by B. T. Pickering (13
November, 288, 117; 1980) the molecular weights
quoted were incorrectly altered by the insertion
of ' $K$ ' and should instead by read in Daltons.

